\title{
Late complications of nylon suture blepharoplasty causing ocular surface injury
}

\author{
Kazunami Noma, MD, $\mathrm{PhD}^{1}$, Yasuhiro Takahashi, MD, $\mathrm{PhD}^{2}$, Maria Suzanne Sabundayo, $\mathrm{MD}^{2}$, \\ Hirohiko Kakizaki, MD, $\mathrm{PhD}^{2}$ \\ ${ }^{1}$ Noma Eye Clinic, Hiroshima, Japan, ${ }^{2}$ Aichi Medical University Hospital, Nagakute, Japan
}

\begin{abstract}
Background: There are several known ocular complications of suture blepharoplasty which includes suture exposure, cyst formation, and surgical site infection.

Objective: To present late complications of nylon suture blepharoplasty that causes damage to the ocular surface.

Methods: Medical records of patients who developed ocular surface injury at least 1 year after nylon suture blepharoplasty were reviewed.

Results: A total of 9 eyes ( 3 right and 6 left) of 9 patients (all females) were included in this study. All patients underwent trans-tarsal nylon suture blepharoplasty. The average age of the patients was 36.2 years (range: $22-64$ years). The average interval between suture blepharoplasty and revision surgery was 9.6 years (range: $5-15$ years). The mean follow-up period after revision surgery was 17 months (range: 7-48 months). Before revision surgery, all patients had reported ocular discomfort. Slit lamp examination revealed ocular surface injury caused by the presence of granulation tissue in the palpebral conjunctiva and deformity of the tarsal plate. Intraoperatively, suture exposure was absent in all but one eyelid. All of the symptoms completely disappeared postoperatively.

Conclusion: Late complications of nylon suture blepharoplasty such as granulation tissue formation and tarsal plate deformity cause damage to the ocular surface. The trans-tarsal technique and hydrolytic denaturation of nylon sutures were considered as the main contributory factors leading to the mechanisms of ocular surface injury.

Level of Evidence: Level IV, case series
\end{abstract}

Keywords: suture blepharoplasty; ocular surface injury; granulation tissue; nylon; trans-tarsal technique

\section{Introduction}

Suture blepharoplasty is the first line technique for the creation of a double eyelid crease in Asians [1]. However, this procedure has several ocular complications [2]. Since ocular surface injury is mostly caused by suture exposure following the trans-tarsal technique [2,3], this complication can be prevented by a procedure without trans-tarsal suturing [2]. Cystic formation or infection around the suture is another complication [4-6], which is resolved by suture removal and topical medical treatment [6].

We experienced complications leading to ocular surface injury several years after nylon suture blepharoplasty. This included granulation formation on the tarsal plate, possibly caused by hydrolysis of the nylon suture [7], and tarsal plate deformity. Ocular surface injury was not improved by simple suture removal and topical medical treatment, necessitating an additional surgery to correct the tarsal plate deformity. 


\section{Materials and methods}

Medical records of all patients who developed ocular surface injury at least 1 year after suture blepharoplasty were reviewed at Noma Eye Clinic and Aichi Medical University Hospital. These patients were examined between January 2008 and June 2011. The surgeons were asked what kind of suture material was used. Informed consent was obtained from all patients. This study was approved by the Institutional Review Board at Aichi Medical University Hospital and adhered to the tenets of the 1964 Declaration of Helsinki.

\section{Surgical methods}

Revision surgery was performed to relieve the mechanical friction between the ocular surface and deformed tarsal plate.

A horizontal incision was first added on the area of granula-

tion tissue (Fig. 1A). After removing a small amount of tarsal tissue, the granulation tissue was removed without harming the meibomian glands. Any exposed suture material was also removed. Diathermy coagulation was performed on the incision area to flatten the tarsal plate (Fig. 1B). For further tarsal flattening, a couple of short vertical incisions $(2.5 \mathrm{~mm})$ were added on the tarsal plate (Fig. 1C), after which diathermy coagulation was performed (Fig. 1D). After sufficient hemostasis, $0.3 \%$ ofloxacin eye ointment was administered.

\section{Results}

Patient data are shown in Table 1. Nine eyes (3 right and 6 left) from 9 patients (all females) were included in the study. All patients underwent trans-tarsal nylon suture blepharoplasty. The average age of the patients was 36.2 years (range: 22-64
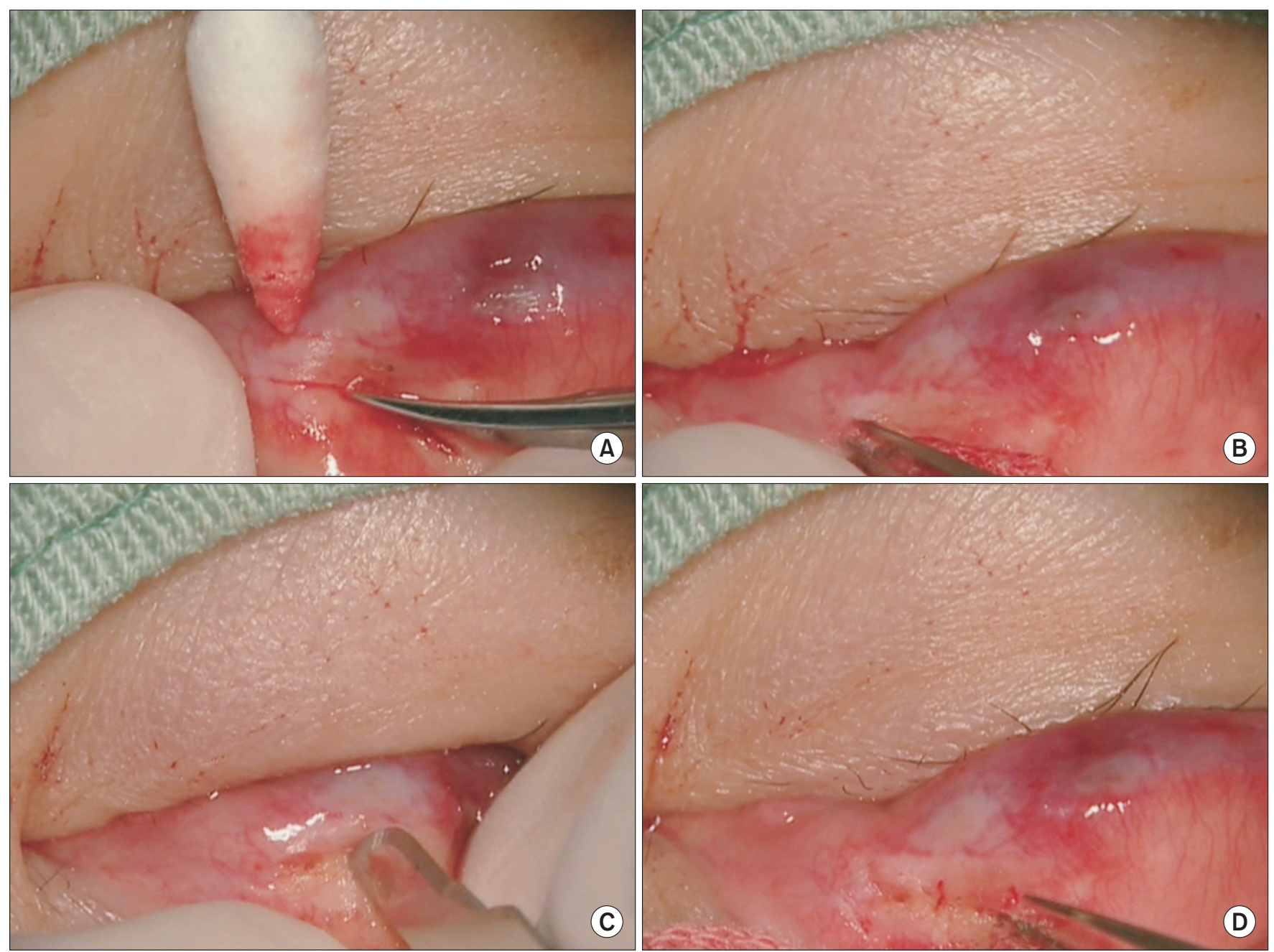

Fig. 1. (A) A horizontal incision was added on the area of granulation. (B) Diathermy coagulation was performed on the incision area for the purpose of flattening the tarsal plate. (C) A couple of short vertical incisions (2.5 $\mathrm{mm}$ each) were added on the tarsal plate for further flattening. (D) Diathermy coagulation was performed on the short vertical incisions. 
Table 1. Patient data

\begin{tabular}{ccccccc}
\hline Case & Sex & Age (year-old) & Side & Duration after SB (years) & Suture material & Follow-up period (months) \\
\hline 1 & F & 28 & R & 5 & Nylon & Nylon \\
2 & F & 33 & L & 13 & Nylon & 7 \\
3 & F & 28 & L & 6 & Nylon & 7 \\
4 & F & 43 & R & 5 & Nylon & 8 \\
\hline 5 & F & 28 & L & 10 & Nylon & 24 \\
6 & F & 22 & L & 6 & Nylon & 29 \\
7 & F & 64 & L & 12 & Nylon & 48 \\
\hline 8 & F & 49 & L & 15 & Nylon & 9 \\
\hline 9 & F & 31 & R & 13 & & \\
\hline
\end{tabular}

F, female; R, right; L, left; SB, suture blepharoplasty.
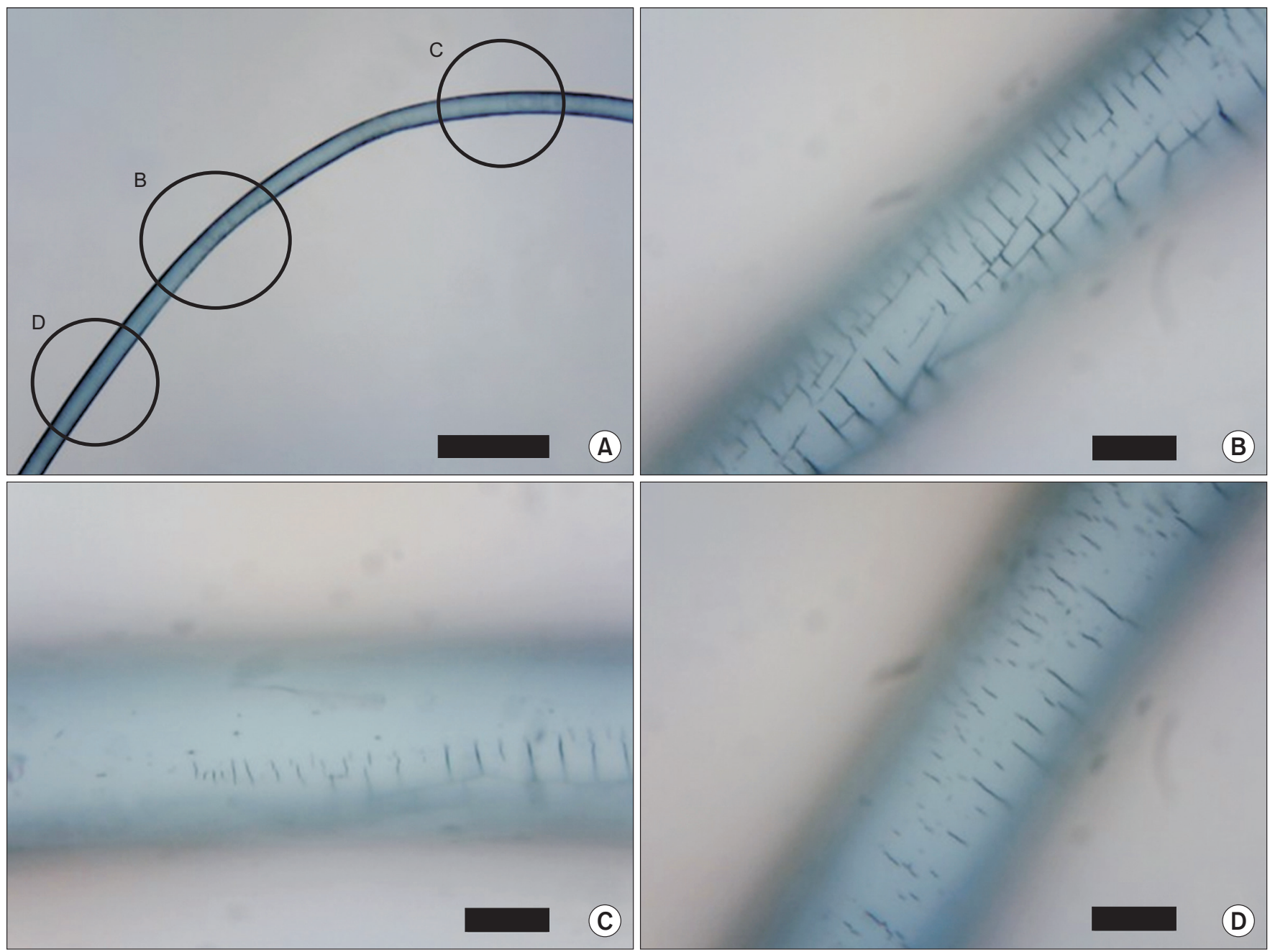

Fig. 2. Light microscopic image of the exposed suture harvested from the case 4. (A) Using low power magnification, a segment of the suture with narrow diameter is seen (area B). Bar=0.3 mm. (B-D) High power figures of areas of B, C and D in Fig. 2A, respectively. Narrow diameter part (B) shows more cracks than stable diameter part (C) and (D). Bar=0.05 mm.

years). The average interval between suture blepharoplasty and revision surgery was 9.6 years (range: $5-15$ years). The mean follow-up period after revision surgery was 17 months (range: 7-48 months).
All patients had reported ocular discomfort prior to revision surgery. Slit lamp examination revealed ocular surface injury caused by the presence of granulation tissue on the palpebral conjunctiva and deformity of the tarsal plate. Intraoperatively, 
suture exposure was absent in all but one eyelid. All these symptoms completely disappeared postoperatively.

The exposed suture was harvested from Case \#4 who underwent suture blepharoplasty 5 years before revision surgery. Slide viewed under light microscopy using low power $(\times 40)$ showed a narrow segment of the suture (area B in Fig. 2A). Figs. 2B, C and D are high-power field images $(\times 400)$ of areas B, C and D in Fig. 2A, respectively. The narrow segment (Fig. 2B) showed more cracks compared to the other areas (Figs. 2C and D).

A representative case is presented below.

\section{Case \#4}

The patient underwent trans-tarsal nylon suture blepharoplasty in 2006. Five years after surgery, ocular discomfort was felt on the right eye. The best-corrected visual acuity was 1.0 on both eyes. On the right eye, the cornea had superficial punctuate keratopathy and the bulbar conjunctiva had epithelial defects (Fig. 3A). Conjunctival injection was severe (Fig. 3B). A suture segment was found exposed from a protruded portion on the nasal aspect of the upper tarsal plate. (Fig. 3C). The exposed suture was subsequently removed. Ophthalmic drops ( $0.5 \%$ levofloxacin and $0.1 \%$ fluorometholone) were prescribed 4 times daily for 1 week; however, the ocular discomfort did not improve. The contralateral eye did not demonstrate any signs despite having undergone the same operation using the same nylon suture (Fig. 3D).
We performed revision surgery on the deformed right tarsal plate. After the first postoperative week, no corneal and conjunctival epithelial defects were observed (Fig. 3E) in spite of the irregular surface of the palpebral conjunctiva (Fig. 3F). Complete resolution of the ocular surface was seen after 2 months (Fig. 3G), irrespective of the conjunctival scar (Fig. 3H). The tarsal plate was flat without conjunctival injection (Fig. $3 \mathrm{H})$. The patient did not complain of any symptoms and had demonstrated a clear ocular surface during the 8 postoperative months.

\section{Discussion}

We reported a series of cases involving granulation tissue formation and tarsal deformity which are late complications of nylon suture blepharoplasty causing ocular surface injury. As this complication occurred several years after surgery and most patients with eye symptoms seek consultation at an eye clinic, cosmetic surgeons are less likely to encounter this condition. This may explain why these complications have seldom been reported. However, these complications present a crucial concern in eyelid cosmetic surgery.

First, the surgical method of suture blepharoplasty should be considered. There are roughly 2 types of suture blepharoplasty techniques: trans-tarsal or trans-levator aponeurosis [2]. All patients in the present study underwent the trans-tarsal
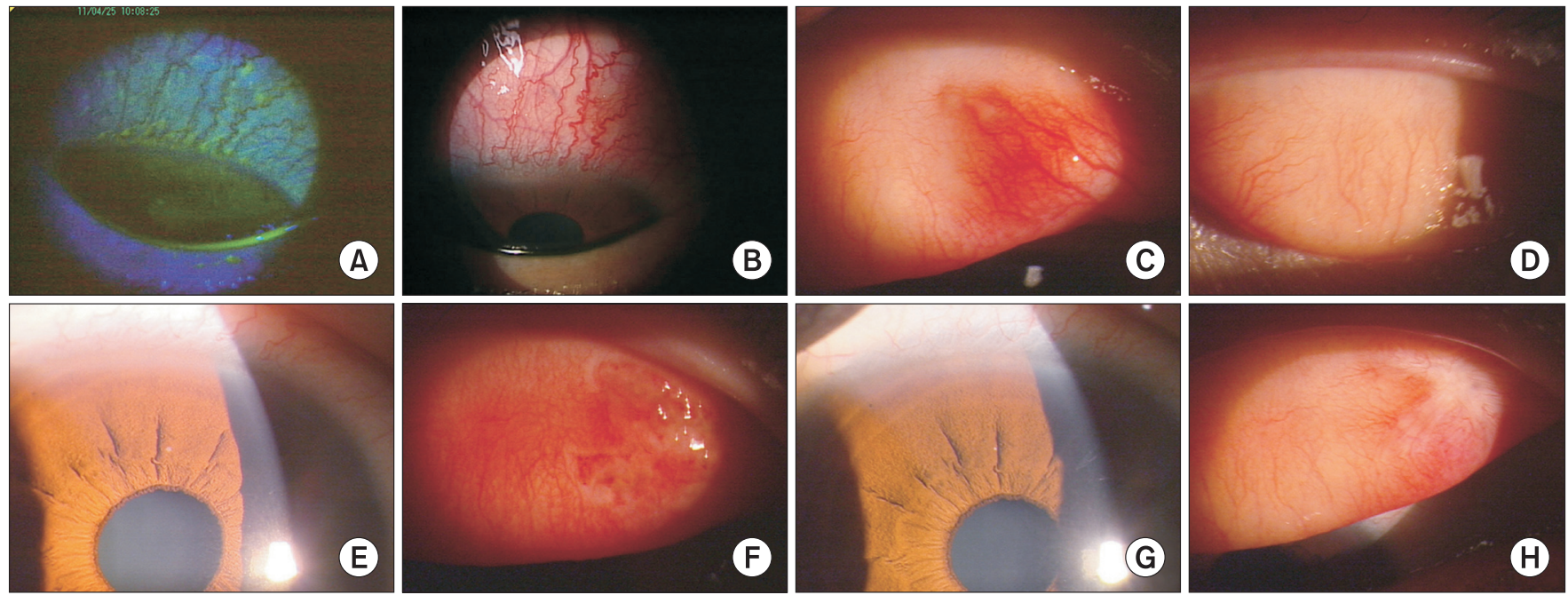

Fig. 3. Photographs of case 4. (A) The right cornea shows superficial punctuate keratopathy and the bulbar conjunctiva also demonstrates epithelial defects. (B) Ocular injection is severe. (C) The palpebral conjunctiva is injected with a suspected focus of inflammation. An exposed suture was found in the tarsal plate but it is obscure in this figure. (D) The contralateral side is unremarkable. (E) There are no corneal and conjunctival epithelial defects one week after the revision surgery. (F) The palpeberal conjunctival surface is still irregular one week after the revision surgery. (G) A clear ocular surface is demonstrated 2 months after the revision surgery. (H) A conjunctival scar is demonstrated 2 months after the revision surgery, but the tarsal plate is flat without conjunctival injection. 
technique which subsequently lead to a deformed suture point that eventually injured the ocular surface. As the trans-levator aponeurosis technique does not suture the tarsal surface but does so above the superior margin of the tarsal plate, the suture area does not touch the ocular surface, avoiding ocular trauma [2]. Therefore, the trans-levator aponeurosis technique is a safer method to protect the ocular surface.

Second, the choice of suture material should be taken into account. Nylon sutures were used in all patients in this study. Nylon has amide bonds and undergoes hydrolysis for a longer time in vivo [7]. The average interval of 9.6 years (range: 5-15 years) between suture blepharoplasty and revision surgery may have been long enough for the nylon suture to undergo hydrolysis. In fact, the suture harvested from Case \#4 showed a narrow segment with many cracks, suggesting a hydrolytic denaturation process [7].

Nylon loses its tensile strength and incites a stronger biological reaction than polypropylene [7] and polyvinylidene [8]. Based on this fact, polypropylene or polyvinylidene is considered the suture material of choice in vascular anastomoses surgery $[7,8]$. As such, the use of polypropylene or polyvinylidene sutures instead of nylon sutures is recommended in suture blepharoplasty to avoid these late complications.

Revision surgery was performed to relieve the mechanical friction between the ocular surface and deformed tarsal plate. Horizontal and vertical incisions were made at the areas of tarsal deformity in order to induce scar formation and thereby flatten the tarsal surface. This technique may initially appear to produce a rougher tarsal surface against the ocular surface; however, ocular surface improvement and absence of foreign body sensation was seen in all patients during the long-term follow-up period.

A previous study reported a similar late-onset complication after trans-tarsal suture blepharoplasty [9]. Patients in this previous study showed chronic palpebral conjunctival inflammation and tarsal plate deformity [9]. However, this study did not mention the effect of the type of suture material and the presence of granulation tissue on the palpebral conjunctiva [9]. In addition, the author did not correct the tarsal plate deformity [9].

All of the patients presented with unilateral and single-point involvement, despite having undergone the same surgery using the same nylon suture on the contralateral side. The reason behind this was unclear; however, factors such as difference in suture tightness and asymmetric ocular surface may have contributed to its unilateral occurrence.

\section{Conclusion}

We reported late complications of nylon suture blepharoplasty causing ocular surface injury. The trans-tarsal technique and hydrolytic denaturation of nylon sutures were considered as the main contributory factors leading to the mechanisms of ocular surface injury.

\section{Conflicts of interest}

The authors have nothing to disclose.

\section{References}

1. Chen WPD. Suture ligation methods. In: Chen WPD, editor. Asian blephraoplasty and the eyelid crease. 2nd ed. Philadelphia: Butterworth-Heinemann; 2006. p. 39-50.

2. Sakai S. [Juuken-jutsu: maibotsu-hou]. In: Nemoto Y, editor. Practical ophthalmology 99: meno Keiseigeka. Tokyo: Bunkodo; 2003. p. 70-4. Japanese.

3. Tsurukiri K. New modified double eyelid technique to avoid corneal damage. J Jpn Aesth Plast Surg 1997;19:87-93.

4. Baek SM, Kim SS, Tokunaga S, Bindiger A. Oriental blepharoplasty: single-stitch, nonincision technique. Plast Reconstr Surg 1989;83:236-42.

5. Choi Y, Eo S. Tissue thread grafting: nonincisional doubleeyelid operation. Ann Plast Surg 2010;64:376-80.

6. Homma K, Mutou Y, Mutou H, Ezoe K, Fujita T. Intradermal stitch blepharoplasty for orientals: does It disappear? Aesthetic Plast Surg 2000;24:289-91.

7. Azuma T, Hirayama T, Misumi Y, Uesugi H, Ideta I. Anastomotic aneurysm due to fragmentation of monofilament polyamide suture (Nylon 6-66). Jpn J Vasc Surg 2007;16:619-23.

8. Laroche G, Marois Y, Schwarz E, Guidoin R, King MW, Pâris E, et al. Polyvinylidene fluoride monofilament sutures: can they be used safely for long-term anastomoses in the thoracic aorta? Artif Organs 1995;19:1190-9.

9. Mizuno T. Treatment of suture-related complications of buriedsuture double-eyelid blepharoplasty in Asians. Plast Reconstr Surg Glob Open 2016;4:e839. 